\title{
Progress in rechargeable aqueous alkali-ion batteries in China
}

\author{
Jinming Yue ${ }^{1,2}$, Liumin Suo $o^{1,2,3^{*}}$
}

1. Beijing Advanced Innovation Center for Materials Genome Engineering, Key Laboratory for Renewable Energy, Beijing Key Laboratory for New Energy Materials and Devices, Beijing National Laboratory for Condensed Matter Physics, Institute of Physics, Chinese Academy of Sciences, Beijing 100190, China.

2. Center of Materials Science and Optoelectronics Engineering, University of Chinese Academy of Sciences, Beijing 100049, China.

3. Yangtze River Delta Physics Research Center Co. Ltd, Liyang, 213300, China.

Email: suoliumin@iphy.ac.cn 
Table S1. The detailed information of the commonly used cathode materials for ALIBs.

\begin{tabular}{|c|c|c|c|c|c|}
\hline Cathode materials for ALIBs & $\begin{array}{l}\text { Capacity } \\
(\mathrm{mAh} / \mathrm{g})\end{array}$ & $\begin{array}{c}\text { Cycle } \\
\text { number }\end{array}$ & $\begin{array}{c}\text { Capacity retention } \\
\text { (rate/current) }\end{array}$ & Electrolyte & Reference \\
\hline $\mathrm{LiNi}_{1 / 3} \mathrm{Co}_{1 / 3} \mathrm{Mn}_{1 / 3} \mathrm{O}_{2}$ & 64 & 1000 & $90.9 \%(80 \mathrm{C})$ & $2 \mathrm{M} \mathrm{LiNO}_{3}$ & 82 \\
\hline $\begin{array}{c}\mathrm{LiNi}_{1 / 3} \mathrm{Co}_{1 / 3} \mathrm{Mn}_{1 / 3} \mathrm{O}_{2} \\
\text { nanoblocks }\end{array}$ & 138 & 20 & $95.2 \%(1 \mathrm{C})$ & $2 \mathrm{M} \mathrm{LiNO}_{3}$ & 83 \\
\hline $\mathrm{LiMnPO}_{4}$ & 60 & 80 & $\sim 80 \%(0.075 \mathrm{~A} / \mathrm{g})$ & $2 \mathrm{M} \mathrm{Li}_{2} \mathrm{SO}_{4}$ & 84 \\
\hline $\mathrm{Li}_{3} \mathrm{~V}_{2}\left(\mathrm{PO}_{4}\right)_{3} / \mathrm{C}$ & 91.6 & 50 & $98.9 \%(20 \mathrm{C})$ & saturated $\mathrm{LiNO}_{3}$ & 57 \\
\hline $\mathrm{Li}_{3} \mathrm{~V}_{2}\left(\mathrm{PO}_{4}\right)_{3} / \mathrm{C}$ & 100 & 50 & $68.8 \%(5 C)$ & $\begin{array}{c}\text { saturated } \mathrm{LiNO}_{3} \\
\mathrm{pH}=5\end{array}$ & 85 \\
\hline \multirow[t]{3}{*}{ porous $\mathrm{LiMn}_{2} \mathrm{O}_{4}$} & 112 & 10000 & $93 \%(9 C)$ & $0.5 \mathrm{M} \mathrm{Li}_{2} \mathrm{SO}_{4}$ & 43 \\
\hline & 114 & 400 & $69.3 \%(0.5 \mathrm{~A} / \mathrm{g})$ & $2 \mathrm{M} \mathrm{Li}_{2} \mathrm{SO}_{4}$ & \multirow{4}{*}{86} \\
\hline & 108 & 500 & $68.8 \%(0.5 \mathrm{~A} / \mathrm{g})$ & $1 \mathrm{M} \mathrm{LiNO}_{3}$ & \\
\hline \multirow[t]{2}{*}{$\mathrm{LiMn}_{2} \mathrm{O}_{4}$} & 119 & 1500 & $71.2 \%(1 \mathrm{~A} / \mathrm{g})$ & $5 \mathrm{M} \mathrm{LiNO}_{3}$ & \\
\hline & 121 & 400 & $67.2 \%(0.5 \mathrm{~A} / \mathrm{g})$ & $9 \mathrm{M} \mathrm{LiNO}_{3}$ & \\
\hline $\mathrm{LiMn}_{2} \mathrm{O}_{4} / \mathrm{MWNTs}$ & 112.8 & 1000 & $96.41 \%(0.1 \mathrm{~A} / \mathrm{g})$ & $1 \mathrm{M} \mathrm{Li}_{2} \mathrm{SO}_{4}$ & 87 \\
\hline Nanochain $\mathrm{LiMn}_{2} \mathrm{O}_{4}$ & 110 & 200 & $98 \%(0.5 \mathrm{~A} / \mathrm{g})$ & $0.5 \mathrm{M} \mathrm{Li}_{2} \mathrm{SO}_{4}$ & 88 \\
\hline $\mathrm{LiMn}_{2} \mathrm{O}_{4}$ rods & 91.1 & 200 & $94.6 \%(0.5 \mathrm{~A} / \mathrm{g})$ & $1 \mathrm{M} \mathrm{Li}_{2} \mathrm{SO}_{4}$ & 89 \\
\hline $\mathrm{LiMn}_{2} \mathrm{O}_{4}$ Nanotube & 59.3 & $\sim$ & $\sim(600 \mathrm{C})$ & $0.5 \mathrm{M} \mathrm{Li}_{2} \mathrm{SO}_{4}$ & 90 \\
\hline $\mathrm{LiMn}_{2} \mathrm{O}_{4}$ & 96 & 100 & $96 \%(0.2 \mathrm{~A} / \mathrm{g})$ & $5 \mathrm{M} \mathrm{LiNO}_{3}$ & 91 \\
\hline $\mathrm{LiCoO}_{2}$ & 115 & 50 & $91.3 \%(0.025 \mathrm{~A} / \mathrm{g})$ & $2 \mathrm{M} \mathrm{LiNO}_{3}$ & 92 \\
\hline Nano-LiCoO ${ }_{2}$ & 122 & 40 & $\sim 100 \%(7 \mathrm{C})$ & $0.5 \mathrm{M} \mathrm{Li}_{2} \mathrm{SO}_{4}$ & 93 \\
\hline $\mathrm{TiO}_{2}$-coated $\mathrm{LiCoO}_{2}$ & 128 & 100 & $\sim 100 \%(10 \mathrm{~A} / \mathrm{g})$ & $0.5 \mathrm{M} \mathrm{Li}_{2} \mathrm{SO}_{4}$ & 94 \\
\hline $\mathrm{LiCoO}_{2}$ nanosheets & 126 & 1000 & $88 \%(1 \mathrm{C})$ & saturated $\mathrm{Li}_{2} \mathrm{SO}_{4}$ & 95 \\
\hline $\mathrm{LiFePO}_{4} / \mathrm{C} / \mathrm{CNTs}$ & 158 & 60 & $\sim 100 \%(1 \mathrm{C})$ & Saturated $\mathrm{LiNO}_{3}$ & 58 \\
\hline Macro-porous $\mathrm{LiFePO}_{4}$ & 108 & 50 & $\sim 100 \%(0.1 \mathrm{~A} / \mathrm{g})$ & $0.5 \mathrm{M} \mathrm{Li}_{2} \mathrm{SO}_{4}$ & 96 \\
\hline $\mathrm{LiFePO}_{4} / \mathrm{C}$ & 163.5 & 110 & $91.1 \%(0.1 \mathrm{C})$ & $1 \mathrm{M} \mathrm{Li}_{2} \mathrm{SO}_{4}$ & 97 \\
\hline
\end{tabular}


Table S2. The detailed information of the commonly used anode materials for ALIBs.

\begin{tabular}{|c|c|c|c|c|c|}
\hline Anode materials for ALIBs & $\begin{array}{l}\text { Capacity } \\
(\mathrm{mAh} / \mathrm{g})\end{array}$ & $\begin{array}{c}\text { Cycle } \\
\text { number }\end{array}$ & $\begin{array}{l}\text { Capacity retention } \\
\text { (rate/current) }\end{array}$ & Electrolyte & Reference \\
\hline $\mathrm{V}_{2} \mathrm{O}_{5}$ & 136 & 50 & $70 \%(0.1 \mathrm{C})$ & $1 \mathrm{M} \mathrm{Li}_{2} \mathrm{SO}_{4}$ & \multirow{2}{*}{98} \\
\hline $1.6 \% \mathrm{AIPO}_{4}$-coated $\mathrm{V}_{2} \mathrm{O}_{5}$ & 128 & 50 & $99 \%(0.1 \mathrm{C})$ & $1 \mathrm{M} \mathrm{Li}_{2} \mathrm{SO}_{4}$ & \\
\hline $\mathrm{VO}_{2}$ & 150 & 20 & $\sim 83 \%(0.1 \mathrm{~A} / \mathrm{g})$ & 20 m LiTFSI & 99 \\
\hline $\mathrm{TiO}_{2}$ & $\sim 100$ & 40 & $\sim 87 \%(2 \mathrm{C})$ & $3.5 \mathrm{M} \mathrm{LiCl}+0.25 \mathrm{M} \mathrm{Li}_{2} \mathrm{SO}_{4}, \mathrm{pH}=10$ & 100 \\
\hline $\mathrm{Li}_{4} \mathrm{Ti}_{5} \mathrm{O}_{12}$ & 150 & 300 & $68 \%(1 c)$ & $2 \mathrm{~m}$ LiTFSI-94\%PEG-6\% $\mathrm{H}_{2} \mathrm{O}$ & 33 \\
\hline $\mathrm{Li}_{4} \mathrm{Ti}_{5} \mathrm{O}_{12}$ & 132 & 1000 & $81 \%(20 c)$ & $\mathrm{MSM}-\mathrm{LiClO}_{4}-\mathrm{H}_{2} \mathrm{O}$ & 101 \\
\hline $\mathrm{NaV}_{6} \mathrm{O}_{15}$ & 160 & 50 & $57.5 \%(0.1 \mathrm{~A} / \mathrm{g})$ & Saturated $\mathrm{LiNO}_{3}$ & 102 \\
\hline $\mathrm{NaTi}_{2}\left(\mathrm{PO}_{4}\right)_{3}$ & 97 & 50 & $\sim 64 \%(0.2 \mathrm{~A} / \mathrm{g})$ & $1 \mathrm{M} \mathrm{Li}_{2} \mathrm{SO}_{4}$ & 103 \\
\hline $\begin{array}{c}\mathrm{NaTi}_{2}\left(\mathrm{PO}_{4}\right)_{3} @ \text { carbon } \\
\text { nanotube fibers }\end{array}$ & 127.8 & 3000 & $90.3 \%\left(2 \mathrm{~A} / \mathrm{cm}^{3}\right)$ & Saturated $\mathrm{Li}_{2} \mathrm{SO}_{4}$ & 104 \\
\hline $\mathrm{Mo}_{6} \mathrm{~S}_{8} / \mathrm{C}$ & 82 & 20 & $\sim 95 \%(1 \mathrm{C})$ & $21 \mathrm{M}$ LiTFSI & 105 \\
\hline PNTCDA & 115 & 1000 & $95 \%(10 \mathrm{C})$ & $5 \mathrm{M} \mathrm{LiNO}_{3}$ & 106 \\
\hline PNTCDA & 115 & 200 & $\sim 84.3 \%(0.5 \mathrm{~A} / \mathrm{g})$ & $21 \mathrm{~m}$ LiTFSI & 107 \\
\hline S@ppy & 163 & 100 & $85 \%(0.1 \mathrm{~A} / \mathrm{g})$ & $0.5 \mathrm{M} \mathrm{Li}_{2} \mathrm{SO}_{4}$ & 108 \\
\hline PANI & 110.4 & 100 & $81.4 \%(0.075 \mathrm{~A} / \mathrm{g})$ & saturated $\mathrm{LiNO}_{3}$ & 109 \\
\hline $\mathrm{Pl} /$ activated carbon & 87 & 100 & $93 \%(0.2 \mathrm{~A} / \mathrm{g})$ & $5 \mathrm{M} \mathrm{LiNO}_{3}$ & 91 \\
\hline $\mathrm{Li}_{0.95} \mathrm{Ti}_{2}\left(\mathrm{PO}_{4}\right)_{3} / \mathrm{C}$ & 98.6 & 1000 & $75.3 \%(5 \mathrm{C})$ & Saturated $\mathrm{Li}_{2} \mathrm{SO}_{4}$ & 65 \\
\hline carbon-coated $\mathrm{LiTi}_{2}\left(\mathrm{PO}_{4}\right)_{3}$ & 103.1 & 120 & $80.6 \%(1 \mathrm{C})$ & $2 \mathrm{M} \mathrm{Li}_{2} \mathrm{SO}_{4}$ & 110 \\
\hline carbon-coated $\mathrm{LiTi}_{2}\left(\mathrm{PO}_{4}\right)_{3}$ & 115.2 & 1000 & $82.1 \%(2 \mathrm{C})$ & Saturated $\mathrm{Li}_{2} \mathrm{SO}_{4}$ & 111 \\
\hline $\begin{array}{l}\text { core@shell structural } \\
\qquad \mathrm{LiTi}_{2}\left(\mathrm{PO}_{4}\right)_{3} / \mathrm{C}\end{array}$ & 120 & 200 & $94.1 \%(1 \mathrm{C})$ & $5 \mathrm{M} \mathrm{LiNO}_{3}$ & 112 \\
\hline $\mathrm{LiV}_{3} \mathrm{O}_{8}$ nanorod & 72 & 50 & $88 \%(0.02 \mathrm{~A} / \mathrm{g})$ & $0.5 \mathrm{M} \mathrm{Li}_{2} \mathrm{SO}_{4}$ & 113 \\
\hline PPy@LiV ${ }_{3} \mathrm{O}_{8}$ & 95 & 10 & $84 \%\left(0.25 \mathrm{~A} / \mathrm{cm}^{2}\right)$ & $0.5 \mathrm{M} \mathrm{Li}_{2} \mathrm{SO}_{4}$ & 114 \\
\hline $\mathrm{TiP}_{2} \mathrm{O}_{7} /$ expanded graphite & 57 & 1000 & $75 \%(0.5 \mathrm{~A} / \mathrm{g})$ & $1 \mathrm{M} \mathrm{Li}_{2} \mathrm{SO}_{4}$ & 115 \\
\hline $\begin{array}{l}\text { carbon coated } \mathrm{TiP}_{2} \mathrm{O}_{7} \\
\text { nanoporous microsphere }\end{array}$ & 85 & 500 & $90 \%(0.5 \mathrm{~A} / \mathrm{g})$ & $1 \mathrm{M} \mathrm{Li}_{2} \mathrm{SO}_{4}$ & 116 \\
\hline $\mathrm{TiP}_{2} \mathrm{O} 7 @ \mathrm{C} / \mathrm{CNTS}$ & 87 & 500 & $88.93 \%(2 \mathrm{~A} / \mathrm{g})$ & saturated $\mathrm{LiNO}_{3}$ & 117 \\
\hline
\end{tabular}


Table S3. The detailed information of the commonly used cathode materials for ASIBs. 


\begin{tabular}{|c|c|c|c|c|c|}
\hline Cathode materials for ASIBs & $\begin{array}{l}\text { Capacity } \\
(\mathrm{mAh} / \mathrm{g})\end{array}$ & $\begin{array}{l}\text { Cycle } \\
\text { number }\end{array}$ & $\begin{array}{l}\text { Capacity retention } \\
\text { (rate/current) }\end{array}$ & Electrolyte & Reference \\
\hline FeHCF & 103 & 200 & $96.3 \%(10 \mathrm{C})$ & $1 \mathrm{M} \mathrm{Na}_{2} \mathrm{SO}_{4}$ & 118 \\
\hline FeHCF nanocubes & 82 & 100 & $93 \%(2 \mathrm{C})$ & $1 \mathrm{M} \mathrm{NaNO}_{3}$ at $\mathrm{pH}=3$ & 119 \\
\hline $\begin{array}{c}\text { NiHCF@carbon } \\
\text { nanotubes }\end{array}$ & 52 & 10000 & $80 \%(10 \mathrm{~A} / \mathrm{g})$ & $1 \mathrm{M} \mathrm{Na}_{2} \mathrm{SO}_{4}$ & 41 \\
\hline $\mathrm{NiHCF}$ & 63.1 & 1000 & $96.3 \%(10 \mathrm{C})$ & $6 \mathrm{M} \mathrm{NaClO}_{4}$ & 120 \\
\hline Monoclinic NiHCF & 70 & 1000 & $79.3 \%(0.5 \mathrm{~A} / \mathrm{g})$ & $5 \mathrm{M} \mathrm{NaClO}_{4}$ & 121 \\
\hline $\mathrm{MnHCF}$ & 130 & 200 & $\sim 96 \%(1 \mathrm{C})$ & $15 \mathrm{~m} \mathrm{NaClO}_{4}$ & 32 \\
\hline $\mathrm{MnHCF}$ & 140 & 50 & $\sim 97.85 \%(1 \mathrm{C})$ & $\begin{array}{c}9 \mathrm{~m} \mathrm{NaOTF}+ \\
22 \mathrm{~m} \text { TEAOTF }\end{array}$ & 34 \\
\hline \multirow[b]{2}{*}{$\mathrm{Ni}_{0.2} \mathrm{Co}_{0.8} \mathrm{HCF}$} & 110 & 100 & $88 \%(0.1 \mathrm{~A} / \mathrm{g})$ & $5 \mathrm{M} \mathrm{NaSO}_{3} \mathrm{CF}_{3}$ & \multirow[b]{2}{*}{69} \\
\hline & 84 & 100 & $\sim 65 \%(0.1 \mathrm{~A} / \mathrm{g})$ & $\mathrm{Na}_{2} \mathrm{SO}_{4}$ & \\
\hline $\mathrm{Ni}_{0.6} \mathrm{Co}_{0.4} \mathrm{HCF}$ & 85 & 100 & $90 \%(1 \mathrm{C})$ & $6 \mathrm{M} \mathrm{NaClO}_{4}$ & 122 \\
\hline $\mathrm{ZnHCF}$ & 61 & 50 & $\sim 96 \%(2 \mathrm{C})$ & $17 \mathrm{~m} \mathrm{NaClO}_{4}$ & 75 \\
\hline CuHCF & 57 & 500 & $93 \%(5 \mathrm{C})$ & $1 \mathrm{M} \mathrm{Na}_{2} \mathrm{SO}_{4}$ & 123 \\
\hline CuHCF & 100 & 250 & $80 \%(0.3 \mathrm{~A} / \mathrm{g})$ & saturated $\mathrm{NaNO}_{3}$ & 124 \\
\hline $\mathrm{CoHCF}$ & 102 & 800 & $90 \%(5 \mathrm{C})$ & $1 \mathrm{M} \mathrm{Na}_{2} \mathrm{SO}_{4}$ & 125 \\
\hline $\mathrm{CoHCF}$ & 115 & 100 & $\sim 100 \%(2 \mathrm{C})$ & $1 \mathrm{M} \mathrm{Na}_{2} \mathrm{SO}_{4}+1 \mathrm{wt} \% \mathrm{CoSO}_{4}$ & 126 \\
\hline VHCF & 69 & 1000 & $93.1 \%(1 \mathrm{~A} / \mathrm{g})$ & $\mathrm{NaClO}_{4}+\mathrm{PEG}$ & 127 \\
\hline $\mathrm{Na}_{0.44} \mathrm{MnO}_{2}$ & 46 & 200 & $\sim 93 \%(0.2 \mathrm{~A} / \mathrm{g})$ & $1 \mathrm{M} \mathrm{Na}{ }_{2} \mathrm{SO}_{4}$ & 28 \\
\hline $\mathrm{Na}_{0.44} \mathrm{MnO}_{2}$ & 47 & 200 & $85 \%(2 C)$ & $1 \mathrm{M} \mathrm{Na}{ }_{2} \mathrm{SO}_{4}$ & 133 \\
\hline $\mathrm{Na}_{0.44} \mathrm{MnO}_{2}$ & 40 & 100 & $\sim 90 \%(0.05 \mathrm{~A} / \mathrm{g})$ & $1 \mathrm{M} \mathrm{Na}{ }_{2} \mathrm{SO}_{4}$ & 134 \\
\hline $\mathrm{Na}_{3} \mathrm{~V}_{2}\left(\mathrm{PO}_{4}\right)_{3}$ & 110 & 100 & $78 \%(1 \mathrm{C})$ & $\begin{array}{c}9 \mathrm{~m} \mathrm{NaOTF}+ \\
22 \mathrm{~m} \text { TEAOTF }\end{array}$ & 38 \\
\hline $\mathrm{Na}_{3} \mathrm{~V}_{2}\left(\mathrm{PO}_{4}\right)_{3}$ & 70 & 50 & $15 \%(20 \mathrm{C})$ & $1 \mathrm{M} \mathrm{Na}{ }_{2} \mathrm{SO}_{4}$ & 129 \\
\hline $\mathrm{Na}_{0.66} \mathrm{Mn}_{0.66} \mathrm{Ti}_{0.34} \mathrm{O}_{2}$ & 76 & 300 & $89 \%(2 \mathrm{C})$ & $1 \mathrm{M} \mathrm{Na}_{2} \mathrm{SO}_{4}$ & 37 \\
\hline $\mathrm{Na}_{0.5} \mathrm{Mn}_{0.5} \mathrm{Ti}_{0.5} \mathrm{O}_{2}$ & 44 & 100 & $95 \%(0.06 \mathrm{~A} / \mathrm{g})$ & $6 \mathrm{M} \mathrm{NaClO}_{4}$ & 68 \\
\hline $\mathrm{Na}_{2} \mathrm{VTi}\left(\mathrm{PO}_{4}\right)_{3}$ & 51 & 500 & $92 \%(5 \mathrm{C})$ & $1 \mathrm{M} \mathrm{Na}_{2} \mathrm{SO}_{4}$ & 130 \\
\hline $\mathrm{Na}_{2.2} \mathrm{~V}_{1.2} \mathrm{Ti}_{0.8}\left(\mathrm{PO}_{4}\right)_{3} / \mathrm{C}$ & 62 & 100 & $\sim 87 \%(1 \mathrm{C})$ & $6 \mathrm{M} \mathrm{NaClO}_{4}$ & 131 \\
\hline $\mathrm{NaVPO}_{4} \mathrm{~F}$ & 55 & 20 & $\sim 73 \%(0.05 \mathrm{~A} / \mathrm{g})$ & $5 \mathrm{M} \mathrm{NaNO}_{3}$ & 132 \\
\hline $\mathrm{MnO}_{2}$ & 78 & 100 & $\sim 82 \%(5 \mathrm{C})$ & $1 \mathrm{M} \mathrm{Na}_{2} \mathrm{SO}_{4}+2 \mathrm{M} \mathrm{MgSO}_{4}$ & 128 \\
\hline
\end{tabular}


Table S4. The detailed information of the commonly used anode materials for ASIBs.

\begin{tabular}{|c|c|c|c|c|c|}
\hline Anode materials for ASIBs & $\begin{array}{l}\text { Capacity } \\
(\mathrm{mAh} / \mathrm{g})\end{array}$ & $\begin{array}{l}\text { Cycle } \\
\text { number }\end{array}$ & $\begin{array}{l}\text { Capacity retention } \\
\text { (rate/current) }\end{array}$ & Electrolyte & Reference \\
\hline frogspawn-like $\mathrm{NaTi}_{2}\left(\mathrm{PO}_{4}\right)_{3} / \mathrm{C}$ & 110 & 400 & $88 \%(1 \mathrm{C})$ & $1 \mathrm{M} \mathrm{Na}_{2} \mathrm{SO}_{4}$ & 66 \\
\hline $\begin{array}{l}\mathrm{NaTi}_{2}\left(\mathrm{PO}_{4}\right)_{3}- \\
\text { graphene nanosheets }\end{array}$ & 104.4 & 100 & $95.7 \%(2 \mathrm{C})$ & $1 \mathrm{M} \mathrm{Na}_{2} \mathrm{SO}_{4}$ & 76 \\
\hline $\mathrm{NaTi}_{2}\left(\mathrm{PO}_{4}\right)_{3}$ & 100 & 100 & $\sim 80 \%(5 \mathrm{C})$ & $1 \mathrm{M} \mathrm{Na}_{2} \mathrm{SO}_{4}$ & 135 \\
\hline $\begin{array}{c}\text { Wafer-like 3D porous } \\
\text { structured } \mathrm{NaTi}_{2}\left(\mathrm{PO}_{4}\right)_{3} / \mathrm{C}\end{array}$ & 114 & 300 & $82 \%(2 C)$ & $1 \mathrm{M} \mathrm{Na}_{2} \mathrm{SO}_{4}$ & 136 \\
\hline $\mathrm{NaTi}_{2}\left(\mathrm{PO}_{4}\right)_{3} / \mathrm{C}$ & 128 & 50 & $90.4 \%(2 \mathrm{C})$ & $1 \mathrm{M} \mathrm{Na}_{2} \mathrm{SO}_{4}$ & 137 \\
\hline TiN-coated $\mathrm{NaTi}_{2}\left(\mathrm{PO}_{4}\right)_{3}$ & 131.9 & 100 & $70 \%(2 \mathrm{C})$ & $1 \mathrm{M} \mathrm{Na}_{2} \mathrm{SO}_{4}$ & 138 \\
\hline $\begin{array}{l}\text { graphene-integrated } \\
\qquad \mathrm{NaTi}_{2}\left(\mathrm{PO}_{4}\right)_{3} / \mathrm{C}\end{array}$ & 105 & 100 & $92 \%(0.1 \mathrm{~A} / \mathrm{g})$ & $6 \mathrm{M} \mathrm{NaClO}_{4}$ & 139 \\
\hline Polyimide & 166 & 280 & $88.5 \%(3 \mathrm{C})$ & $1 \mathrm{M} \mathrm{Na}_{2} \mathrm{SO}_{4}$ & 41 \\
\hline Polyimide & 165 & 20 & $83 \%(0.05 \mathrm{~A} / \mathrm{g})$ & $5 \mathrm{M} \mathrm{NaNO}{ }_{3}$ & 132 \\
\hline Polyimide-MWCNTs & 120 & 500 & $\sim 83 \%(10 \mathrm{C})$ & $1 \mathrm{M} \mathrm{Na}_{2} \mathrm{SO}_{4}$ & 134 \\
\hline $\mathrm{Na}_{2} \mathrm{VTi}\left(\mathrm{PO}_{4}\right)_{3}$ & 48 & 500 & $94 \%(5 C)$ & $1 \mathrm{M} \mathrm{Na}_{2} \mathrm{SO}_{4}$ & 130 \\
\hline $\mathrm{TiS}_{2}$ & 140 & 200 & $\sim 93 \%$ (1C) & $15 \mathrm{~m} \mathrm{NaClO}_{4}$ & 32 \\
\hline $\mathrm{NaTiOPO}_{4}$ & 53 & 120 & $\sim 56 \%(2 \mathrm{C})$ & $\begin{array}{l}9 \mathrm{~m} \mathrm{NaOTF}+ \\
22 \mathrm{~m} \text { TEAOTF }\end{array}$ & 34 \\
\hline $\mathrm{Na}_{0.44} \mathrm{Mn}_{0.44} \mathrm{Ti}_{0.56} \mathrm{O}_{2}$ & 40 & 400 & $\sim 98 \%(2 \mathrm{C})$ & $\mathrm{Na}_{2} \mathrm{SO}_{4} \mathrm{pH}=13.5$ & 36 \\
\hline PNTCDA & 110 & 50000 & $70 \%(5 \mathrm{~A} / \mathrm{g})$ & $\begin{array}{c}0.1 \mathrm{M} \mathrm{Nal}+0.01 \mathrm{M} \mathrm{I}_{2}+1 \mathrm{M} \\
\mathrm{NaNO}_{3}\end{array}$ & 140 \\
\hline $\mathrm{Na}_{2} \mathrm{~V}_{6} \mathrm{O}_{16}$ & 45 & 50 & $\sim 73 \%(0.04 \mathrm{~A} / \mathrm{g})$ & $1 \mathrm{M} \mathrm{Na}_{2} \mathrm{SO}_{4}$ & 141 \\
\hline ppy@MoO 3 & 33 & 1000 & $\sim 87 \%(0.55 \mathrm{~A} / \mathrm{g})$ & $0.5 \mathrm{M} \mathrm{Na}_{2} \mathrm{SO}_{4}$ & 142 \\
\hline
\end{tabular}

Table S5. The detailed information of the commonly used cathode materials for

AKIBs.

\begin{tabular}{|c|c|c|c|c|c|}
\hline Cathode materials for AKIBs & $\begin{array}{l}\text { Capacity } \\
(\mathrm{mAh} / \mathrm{g})\end{array}$ & $\begin{array}{l}\text { Cycle } \\
\text { number }\end{array}$ & $\begin{array}{l}\text { Capacity retention } \\
\text { (rate/current) }\end{array}$ & Electrolyte & Reference \\
\hline FeHCF & 108.6 & 300 & $\sim 92 \%(2 \mathrm{C})$ & saturated $\mathrm{KNO}_{3}$ & 143 \\
\hline $\mathrm{K}_{1.93} \mathrm{Fe}\left[\mathrm{Fe}(\mathrm{CN})_{6}\right]_{0.97} \cdot 1.82 \mathrm{H}_{2} \mathrm{O}$ & 100 & 300 & $88 \%(10 \mathrm{C})$ & $1 \mathrm{M} \mathrm{KNO}_{3}$ & 144 \\
\hline $\mathrm{KFHCF}$ & 100 & 50 & $102 \%(0.2 \mathrm{~A} / \mathrm{g})$ & $30 \mathrm{~m} \mathrm{KFSI}$ & 145 \\
\hline KFeMnHCF & 94 & 10000 & $90 \%(100 \mathrm{C})$ & $22 \mathrm{M} \mathrm{KCF}_{3} \mathrm{SO}_{3}$ & 35 \\
\hline Potassium prussian blue & 74 & 2000 & $\sim 70 \%(2 \mathrm{~A} / \mathrm{g})$ & $2 \mathrm{M} \mathrm{NaNO}_{3} / 60.0$ wt $\%$ maltose & 80 \\
\hline CuHCF & 54 & 100 & $\sim 83 \%(1.5 \mathrm{C})$ & $\mathrm{Na}_{2} \mathrm{SO}_{4} / \mathrm{K}_{2} \mathrm{SO}_{4}$ & 81 \\
\hline
\end{tabular}


Table S6. The detailed information of the commonly used anode materials for

AKIBs.

\begin{tabular}{cccccc}
\hline Anode materials for AKIBs & $\begin{array}{c}\text { Capacity } \\
\text { (mAh/g) }\end{array}$ & $\begin{array}{c}\text { Cycle } \\
\text { number }\end{array}$ & $\begin{array}{c}\text { Capacity retention } \\
\text { (rate/current) }\end{array}$ & Electrolyte & Reference \\
\hline $\mathrm{K}_{\mathrm{x}} \mathrm{V}_{2} \mathrm{O}_{5}$ & 100 & 10000 & $80 \%(0.5 \mathrm{~V} / \mathrm{s})$ & $0.5 \mathrm{M} \mathrm{K}_{2} \mathrm{SO}_{4}$ & 27 \\
$\mathrm{PTCDI}$ & 110 & 1000 & $77 \%(20 \mathrm{C})$ & $22 \mathrm{M} \mathrm{KCF}_{3} \mathrm{SO}_{3}$ & 35 \\
PNTCDA & 140 & 300 & $\sim 76 \%(2 \mathrm{C})$ & saturated KNO & 143 \\
$\mathrm{PTCDA}$ & 101 & 500 & $82 \%(2 \mathrm{~A} / \mathrm{g})$ & $30 \mathrm{~m} \mathrm{KFSI}$ & 145 \\
$\mathrm{KTi}_{2}\left(\mathrm{PO}_{4}\right)_{3}$ & 20 & $\sim$ & $\sim$ & $40 \mathrm{M} \mathrm{HCOOK}_{3}$ & 146 \\
$\mathrm{~K}_{0.36}\left(\mathrm{H}_{2} \mathrm{O}\right) \mathrm{yWS}_{2}$ & 40 & 250 & $50 \%(0.5 \mathrm{~A} / \mathrm{g})$ & $0.5 \mathrm{M} \mathrm{K}_{2} \mathrm{SO}_{4}$ & 147 \\
$\mathrm{~K}_{0.38}\left(\mathrm{H}_{2} \mathrm{O}_{0.82} \mathrm{MoS}_{2}\right.$ & 68 & 50 & $\sim 60 \%(0.5 \mathrm{~A} / \mathrm{g})$ & $1 \mathrm{M} \mathrm{K}_{2} \mathrm{SO}_{4}$ & 148 \\
\hline
\end{tabular}

